\title{
Growth of Trichoderma spp on Different Solid Substrates
}

\author{
B. Boblina*, S. K. Beura, M. K. Mishra and A. G. Panda \\ Department of Plant Pathology, College of Agriculture, Orissa University of Agriculture and \\ Technology, Bhubaneswar, Odisha-751003, India \\ *Corresponding author
}

\section{A B S T R A C T}

\section{Keywords}

Rhizoctonia solani, ecosystem, disease management

Article Info

Accepted:

24 August 2019

Available Online:

10September 2019
Trichoderma is one of the common fungal biocontrol agents being used worldwide for efficient management of various foliar and soil borne plant pathogens. Unlike chemicals, the establishment of these biocontrol agents in the targeted niche requires support even after their application. Two Trichoderma species (Isolate 2 and Isolate 5) were isolated from paddy and groundnut rhizospheres respectively. These two isolates were the two most vigorous ones among all the seven Trichoderma isolates obtained from different crop rhizospheres on the basis of their growth in different media and their inhibitory effects on two potential soil borne pathogens namely Rhizoctonia solani and Sclerotium rolfsii as observed in dual culture experiment. Present study deals with evaluation of eighteen solid substrates. Colony forming units were counted following the serial dilution technique and a gradual decrease was observed over a period of six months. Among different solid substrates tested, vermicompost was observed to be the best solid substrate for isolate 2 recording the maximum cfu count $463.67 \times 10^{7} \mathrm{cfu} / \mathrm{g}$ of substrate in the first month after inoculation and it retained spore viability even after six months of inoculation giving a fairly good cfu count of $97.33 \times 10^{7} \mathrm{cfu} / \mathrm{g}$. For isolate 5 , groundnut shell performed the best, recording the maximum cfu count of $467.67 \times 10^{7} \mathrm{cfu} / \mathrm{g}$ in the first month and $144.00 \times 10^{7}$ $\mathrm{cfu} / \mathrm{g}$ was retained even in the sixth month after inoculation.

\section{Introduction}

Plant diseases are one of the major concerns in cultivation worldwide, consequential in loss of billions of dollars of farm produce. In disease management, the amplified use of chemicals have caused negative impact on environmental quality and resulted in upward trend of many living forms which are resistant to the chemicals (Kumar and Gupta, 2012). Under this changing agriculture scenario, the only technology that seems promising to manage the diseases without disturbing the equilibrium of harmful and useful composition of environment and ecosystem is the use of more and more biological control agents. As we approach the $21 \mathrm{st}$ century, there is an enlarging stress on organically produced food, conservation of biodiversity, unpolluted environment and sustainable agriculture. To compound these challenges, biocontrol agents and biopesticides have emerged as viable alternative in pest and disease management. The genus Trichoderma consists of 
anamorphic group isolated primarily from soil and decomposing organic matter with teleomorphs, when known, belonging to the division Ascomycota (Order Hypocreales). Fungal species belonging to this genus are worldwide in occurrence and easily isolated from soil, decaying wood and other plant organic matter. Trichoderma isolates are characterized by a rapid growth rate in culture and by the production of numerous spores (conidia) with varying shades of green.

Development of formulations with increased shelf life and broad spectrum of action with consistent performance under field conditions could pave the way for commercialization of the technology at a faster rate.

Mainly focus should be on formulation development, mass production, quality aspect, delivery system and its scope in commercialization in India for the management of plant diseases. Term solid state fermentation (SSF) is applied for the processes in which insoluble materials in water is used for the microbial growth. Solidstate fermentation (SSF) is an effective method for the mass production of fungal biopesticides since it provides micropropagules with higher conidia content. Various cheap cereal grains like sorghum, millets and ragi are used as substrates (Lewis, 1991; Jeyarajan, 2006).

The grains are moistened, sterilized and inoculated with Trichoderma and incubated for 10 to 15 days for production of Trichoderma which produces dark green spore coating on the grains. These grains can be powdered finely and used as seed treatment or the grains can be used as it is enriching with FYM for soil application.

Shelf life of the formulations decides the commercialization of biocontrol agents. Formulations should sustain the viable nature of the product for the increased period of storage. Bio control product should have the minimum shelf life of 8 to 12 months for industrialization.

\section{Materials and Methods}

Seven isolates of Trichoderma were collected from different crop rhizospheres through serial dilution of soil. The isolates were identified through their colony colour, structure of their phialides and spores. They were maintained by sub culturing o $\mathrm{n}$ Potato dextrose agar.Two isolates namely Trichoderma Isolate 2 and Trichoderma Isolate 5 were selected from all the collected isolates basing on their growth habit on different media and antagonistic nature against two soil borne pathogens i.e Rhizoctonia sp and Fusarium sp..Eighteen solid substrates were collected from different sources and evaluated against these two isolates. Three replications were maintained for each treatment. Bottles used for spawn production (Mushroom seed) were used for carrying out mass multiplication of Trichoderma. Grains were first washed properly and then half boiled in water depending on their size and thickness of seed coat. Excess water was drained out and the grains were shade dried to retain $60 \%$ moisture approximately. After drying, grains were mixed properly with $\mathrm{CaCO}_{3}$ (Calcium carbonate) powder @30g $\mathrm{CaCO}_{3}$ per $\mathrm{kg}$ of grain. All other solid substrates except grains were filled in the bottles and sterile water was added in order to keep $60 \%$ moisture approximately. These bottles were then plugged with non-absorbent cotton.

The bottles already filled with substrates were steam sterilised in an autoclave at $121.6^{\circ} \mathrm{C}(15$ p.s.i) for about 20 minutes. Previously collected isolates were inoculated into PDA plates by hyphal tip method. The inoculated petriplates were incubated for 10-12 days at $26.5^{\circ} \mathrm{C}$. Discs of $10 \mathrm{~mm}$ diameter were cut from these petriplates using a cork borer. Bottles were then inoculated in aseptic 
condition@ @ 1 disc / bottle (Figure 1 and 2).

Antibiotic was added to the sterilised potato dextrose rose bengal agar medium @ 250 mg/l and was poured into glass petridishes. After a month of inoculation, the entire content of the bottle was mixed thoroughly using a sterile glass rod and was serially diluted to get the final dilution of $10^{-7}$. One millilitre from this solution was taken and spread over the petriplates containing medium. This entire procedure was repeated at monthly intervals up to $6^{\text {th }}$ month for cfu count. The inoculated petriplates were incubated at $27 \pm 1^{0} \mathrm{C}$ for atleast three days in a BOD incubator. The colonies formed were marked with a permanent marker and observation was noted.

\section{Results and Discussion}

All the eighteen substrates were found potent enough for mass multiplication of Trichoderma isolates. The two most virulent Trichoderma isolates were mass multiplied in the substrates and the cfu counts were taken at $10^{-7}$ dilution upto six months at monthly intervals. For Isolate 2, Vermicompost recorded the maximum cfu count $463.67 \times 10^{7}$ $\mathrm{cfu} / \mathrm{g}$ in second month of inoculation followed by $427.67 \times 10^{7} \mathrm{cfu} / \mathrm{g}$ and $366.33 \times 10^{7} \mathrm{cfu} / \mathrm{g}$ in first month and third month respectively and minimum $97.33 \times 10^{7} \mathrm{cfu} / \mathrm{g}$ was registered in the sixth month (Table-1, Figure $3 a \& 3 b$ ).

Table.1 Mean monthly cfu in solid substrates (Isolate 2)

\begin{tabular}{|c|c|c|c|c|c|c|c|}
\hline \multirow[t]{2}{*}{ Sl.No } & \multirow[t]{2}{*}{ Treatments } & $1^{\text {st }}$ month & $2^{\text {nd }}$ month & $3^{\text {nd }}$ month & $4^{\text {th }}$ month & $5^{\text {th }}$ month & $6^{\text {th }}$ month \\
\hline & & $\begin{array}{l}(\mathrm{cfu} \\
\left.\mathrm{x} 10^{7}\right) / \mathrm{g}\end{array}$ & $\left(\mathrm{cfu} \times 10^{7}\right) / \mathrm{g}$ & $\left(\mathrm{cfu} \times 10^{7}\right) / \mathrm{g}$ & $\left(\mathrm{cfu} \times 10^{7}\right) / \mathrm{g}$ & $\left(\mathrm{cfu} \times 10^{7}\right) / \mathrm{g}$ & $\left(\mathrm{cfu} \times 10^{7}\right) / \mathrm{g}$ \\
\hline $\mathbf{1}$ & Spent maize cob & $\begin{array}{l}118.00 \\
(9.07)^{*}\end{array}$ & $\begin{array}{l}99.00 \\
(9.00)\end{array}$ & $\begin{array}{l}83.33 \\
(8.92) \\
\end{array}$ & $\begin{array}{l}45.67 \\
(8.66)\end{array}$ & $\begin{array}{l}39.33 \\
(8.59) \\
\end{array}$ & $\begin{array}{l}34.67 \\
(8.53) \\
\end{array}$ \\
\hline 2 & Maize grain & $\begin{array}{l}118.00 \\
(9.07)\end{array}$ & $\begin{array}{l}124.33 \\
(9.09)\end{array}$ & $\begin{array}{l}94.67 \\
(8.98)\end{array}$ & $\begin{array}{l}47.33 \\
(8.67)\end{array}$ & $\begin{array}{l}40.67 \\
(8.61)\end{array}$ & $\begin{array}{l}18.33 \\
(8.26)\end{array}$ \\
\hline 3 & Wheat grain & $\begin{array}{l}255.67 \\
(9.40)\end{array}$ & $\begin{array}{l}167.67 \\
(9.22)\end{array}$ & $\begin{array}{l}98.33 \\
(8.99)\end{array}$ & $\begin{array}{l}64.33 \\
(8.81)\end{array}$ & $\begin{array}{l}42.33 \\
(8.62)\end{array}$ & $\begin{array}{l}32.00 \\
(8.50)\end{array}$ \\
\hline 4 & Finger millet grain & $\begin{array}{l}133.67 \\
(9.12)\end{array}$ & $\begin{array}{l}103.33 \\
(9.01)\end{array}$ & $\begin{array}{l}79.33 \\
(8.90)\end{array}$ & $\begin{array}{l}66.00 \\
(8.82)\end{array}$ & $\begin{array}{l}38.33 \\
(8.58)\end{array}$ & $\begin{array}{l}16.67 \\
(8.20)\end{array}$ \\
\hline 5 & Rice bran & $\begin{array}{l}272.00 \\
(9.44)\end{array}$ & $\begin{array}{l}137.00 \\
(9.14)\end{array}$ & $\begin{array}{l}121.67 \\
(9.08)\end{array}$ & $\begin{array}{l}104.33 \\
(9.09)\end{array}$ & $\begin{array}{l}88.33 \\
(8.95)\end{array}$ & $\begin{array}{l}70.67 \\
(8.85)\end{array}$ \\
\hline 6 & Rice husk & $\begin{array}{l}333.70 \\
(9.52)\end{array}$ & $\begin{array}{l}251.70 \\
(9.40)\end{array}$ & $\begin{array}{l}210.33 \\
(9.32)\end{array}$ & $\begin{array}{l}174.33 \\
(9.24)\end{array}$ & $\begin{array}{l}119.33 \\
(9.08)\end{array}$ & $\begin{array}{l}90.33 \\
(8.96)\end{array}$ \\
\hline 7 & Cotton Waste & $\begin{array}{l}12.67 \\
(8.09)\end{array}$ & $\begin{array}{l}83.00 \\
(8.92)\end{array}$ & $\begin{array}{l}32.00 \\
(8.51)\end{array}$ & $\begin{array}{l}22.00 \\
(8.34)\end{array}$ & $\begin{array}{l}16.00 \\
(8.20)\end{array}$ & $\begin{array}{l}9.33 \\
(7.97)\end{array}$ \\
\hline 8 & Farm yard manure & $\begin{array}{l}252.33 \\
(9.40)\end{array}$ & $\begin{array}{l}139.33 \\
(9.14)\end{array}$ & $\begin{array}{l}98.33 \\
(8.99)\end{array}$ & $\begin{array}{l}66.33 \\
(8.82)\end{array}$ & $\begin{array}{l}47.00 \\
(8.67)\end{array}$ & $\begin{array}{l}30.00 \\
(8.48)\end{array}$ \\
\hline 9 & Vermi-compost & $\begin{array}{l}427.70 \\
(9.63)\end{array}$ & $\begin{array}{l}463.67 \\
(9.67)\end{array}$ & $\begin{array}{l}366.33 \\
(9.56)\end{array}$ & $\begin{array}{l}181.00 \\
(9.26)\end{array}$ & $\begin{array}{l}137.00 \\
(9.14)\end{array}$ & $\begin{array}{l}97.33 \\
(8.99)\end{array}$ \\
\hline 10 & Sorghum grain & $\begin{array}{l}130.00 \\
(9.11)\end{array}$ & $\begin{array}{l}88.70 \\
(8.95)\end{array}$ & $\begin{array}{l}60.00 \\
(8.78)\end{array}$ & $\begin{array}{l}46.33 \\
(8.66)\end{array}$ & $\begin{array}{l}34.00 \\
(8.53)\end{array}$ & $\begin{array}{l}25.33 \\
(8.40)\end{array}$ \\
\hline 11 & Groundnut Shell & $\begin{array}{l}252.70 \\
(9.40)\end{array}$ & $\begin{array}{l}261.33 \\
(9.42)\end{array}$ & $\begin{array}{l}201.67 \\
(9.30)\end{array}$ & $\begin{array}{l}172.33 \\
(9.24)\end{array}$ & $\begin{array}{l}117.67 \\
(9.07)\end{array}$ & $\begin{array}{l}87.67 \\
(8.94)\end{array}$ \\
\hline 12 & Bagasse & $\begin{array}{l}52.00 \\
(8.72)\end{array}$ & $\begin{array}{l}39.67 \\
(8.60)\end{array}$ & $\begin{array}{l}29.33 \\
(8.47)\end{array}$ & $\begin{array}{l}21.33 \\
(8.33)\end{array}$ & $\begin{array}{l}13.67 \\
(8.13)\end{array}$ & $\begin{array}{l}8.67 \\
(7.94)\end{array}$ \\
\hline 13 & Coir pith & $\begin{array}{l}23.33 \\
(8.37)\end{array}$ & $\begin{array}{l}29.33 \\
(8.47)\end{array}$ & $\begin{array}{l}23.00 \\
(8.36)\end{array}$ & $\begin{array}{l}14.33 \\
(8.16)\end{array}$ & $\begin{array}{l}9.67 \\
(7.98)\end{array}$ & $\begin{array}{l}5.33 \\
(7.73)\end{array}$ \\
\hline 14 & Charcoal & $\begin{array}{l}21.00 \\
(8.32)\end{array}$ & $\begin{array}{l}21.67 \\
(8.34)\end{array}$ & $\begin{array}{l}20.00 \\
(8.30)\end{array}$ & $\begin{array}{l}13.67 \\
(8.13)\end{array}$ & $\begin{array}{l}9.33 \\
(7.97)\end{array}$ & $\begin{array}{l}4.00 \\
(7.59)\end{array}$ \\
\hline 15 & Saw dust & $\begin{array}{l}29.33 \\
(8.47)\end{array}$ & $\begin{array}{l}26.70 \\
(8.43)\end{array}$ & $\begin{array}{l}23.33 \\
(8.37)\end{array}$ & $\begin{array}{l}17.33 \\
(8.24)\end{array}$ & $\begin{array}{l}11.70 \\
(8.06)\end{array}$ & $\begin{array}{l}7.33 \\
(7.86)\end{array}$ \\
\hline 16 & Spent mushroom substrate & $\begin{array}{l}115.67 \\
(9.06)\end{array}$ & $\begin{array}{l}90.33 \\
(8.96)\end{array}$ & $\begin{array}{l}78.67 \\
(8.90)\end{array}$ & $\begin{array}{l}55.33 \\
(8.74)\end{array}$ & $\begin{array}{l}35.67 \\
(8.55)\end{array}$ & $\begin{array}{l}25.00 \\
(8.40)\end{array}$ \\
\hline
\end{tabular}


Int.J.Curr.Microbiol.App.Sci (2019) 8(9): 2519-2529

\begin{tabular}{|l|l|l|l|l|l|l|l|}
\hline $\mathbf{1 7}$ & Pigeonpea husk & 66.00 & 58.33 & 46.33 & 30.67 & 23.00 & 18.33 \\
& & $(8.81)$ & $(8.76)$ & $(8.67)$ & $(8.49)$ & $(8.36)$ & $(8.26)$ \\
\hline 18 & Organic waste & 21.67 & 47.67 & 40.33 & 24.00 & 18.67 & 13.67 \\
& & $(8.33)$ & $(8.68)$ & $(8.60)$ & $(8.38)$ & $(8.27)$ & $(8.13)$ \\
\hline & SE(m) \pm & 0.03 & 0.02 & 0.02 & 0.02 & 0.03 & 0.04 \\
\hline & C.D(0.05) & 0.07 & 0.06 & 0.06 & 0.07 & 0.09 & 0.11 \\
\hline
\end{tabular}

*Figures in the parenthesis are $\log _{10}$ transformed value

Table.2 Mean monthly cfu in solid substrates (Isolate 5)

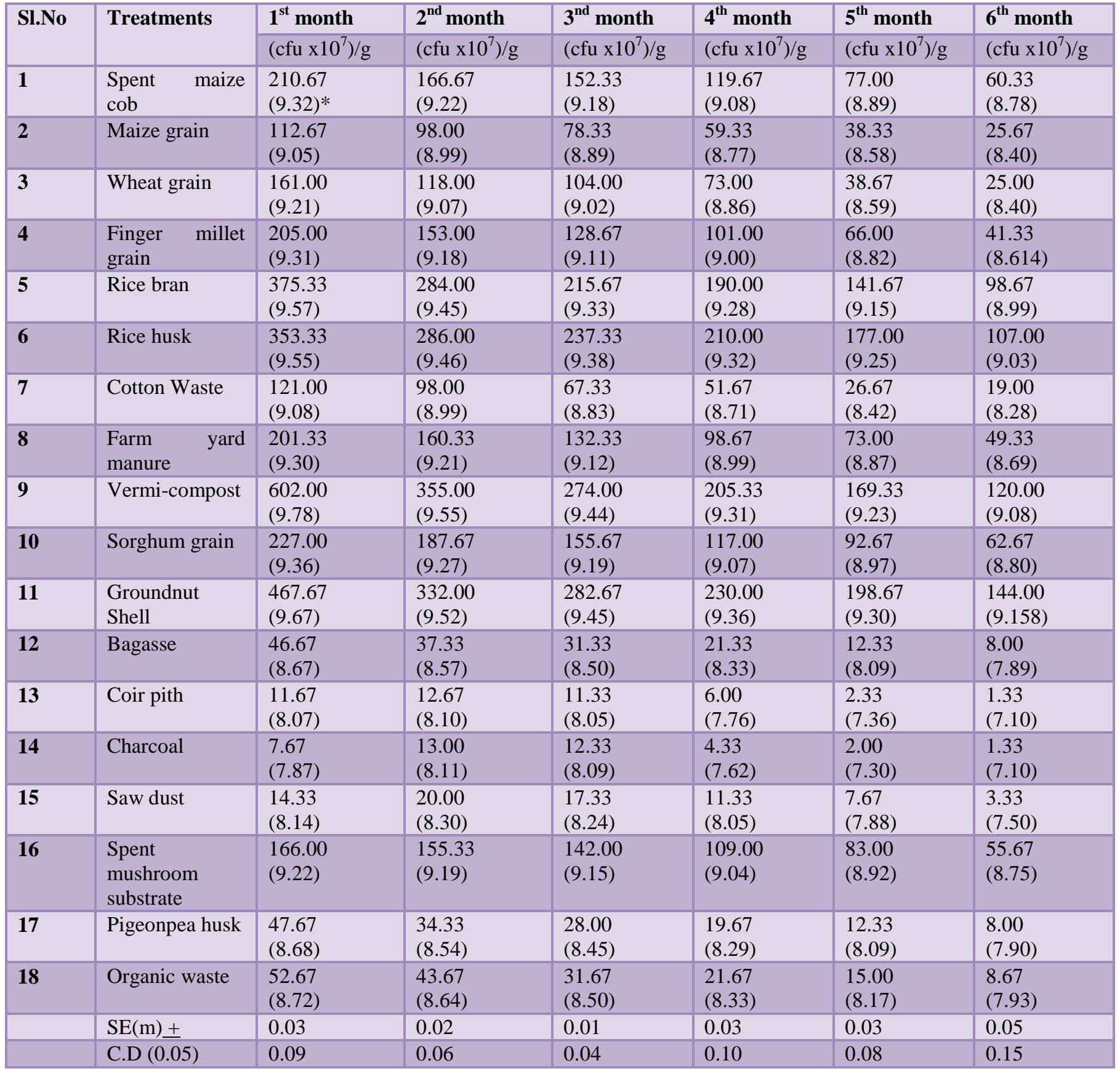

*Figures in the parenthesis are $\log _{10}$ transformed value 


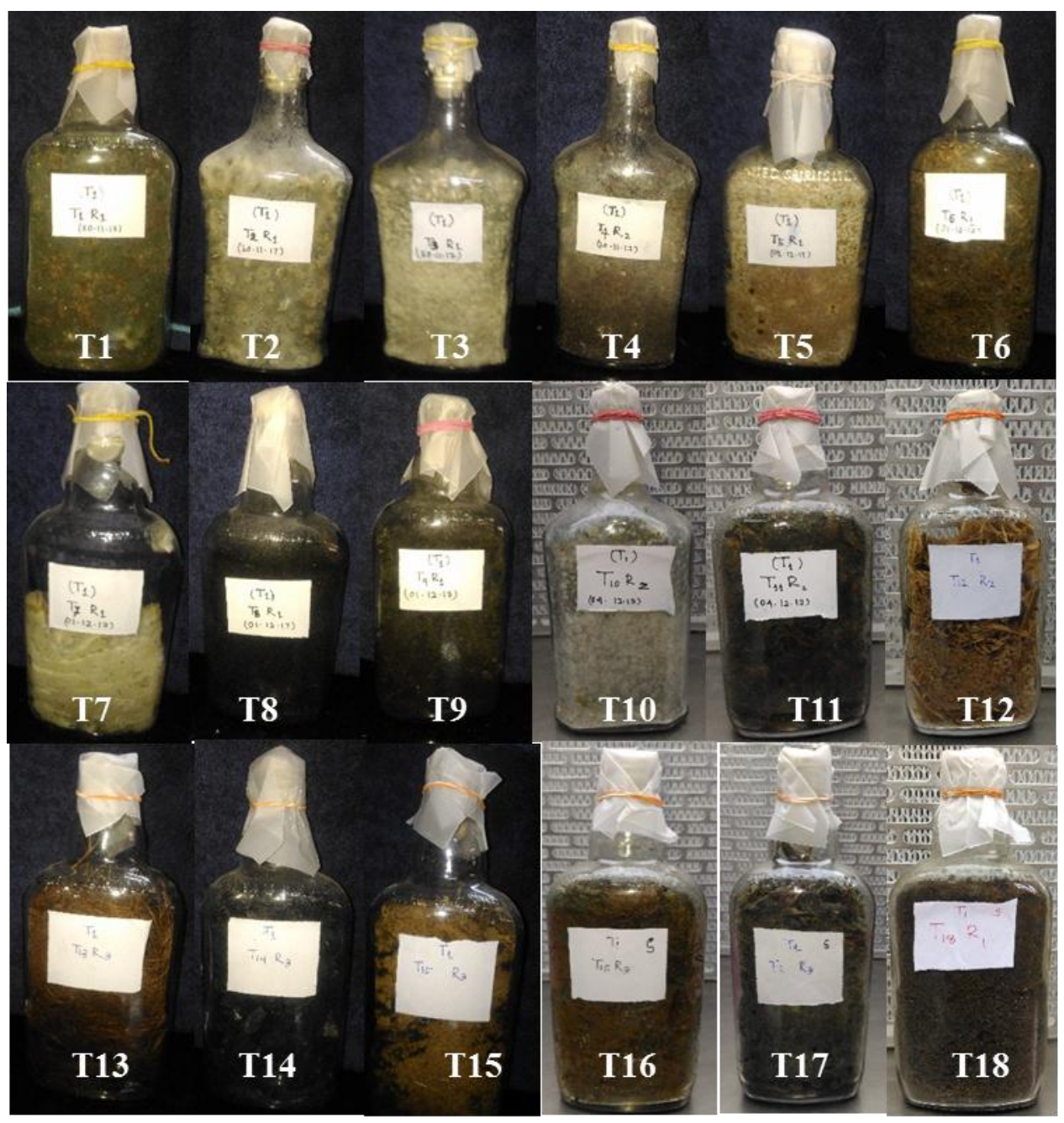

T1: Spent maize cob

T2: Maize grian

T3: Wheat grain

T4: Ragi grain

T5: Rice bran

T6: Paddy husk

T7: Cotton waste

T8: Farm yard Manure

T9: Vermicompost
T10: Sorghum grain

T11: Groundnut shell

T12: Sugarcane bagasse

T13: Coir pith

T14: Charcoal

T15: Saw dust

T16: Spent mushroom substrate T17: Pigeon pea husk

T18: Organic waste

Figure 1- Bottles containing different solid substrates incolulated with Trichoderma Isolate 2 


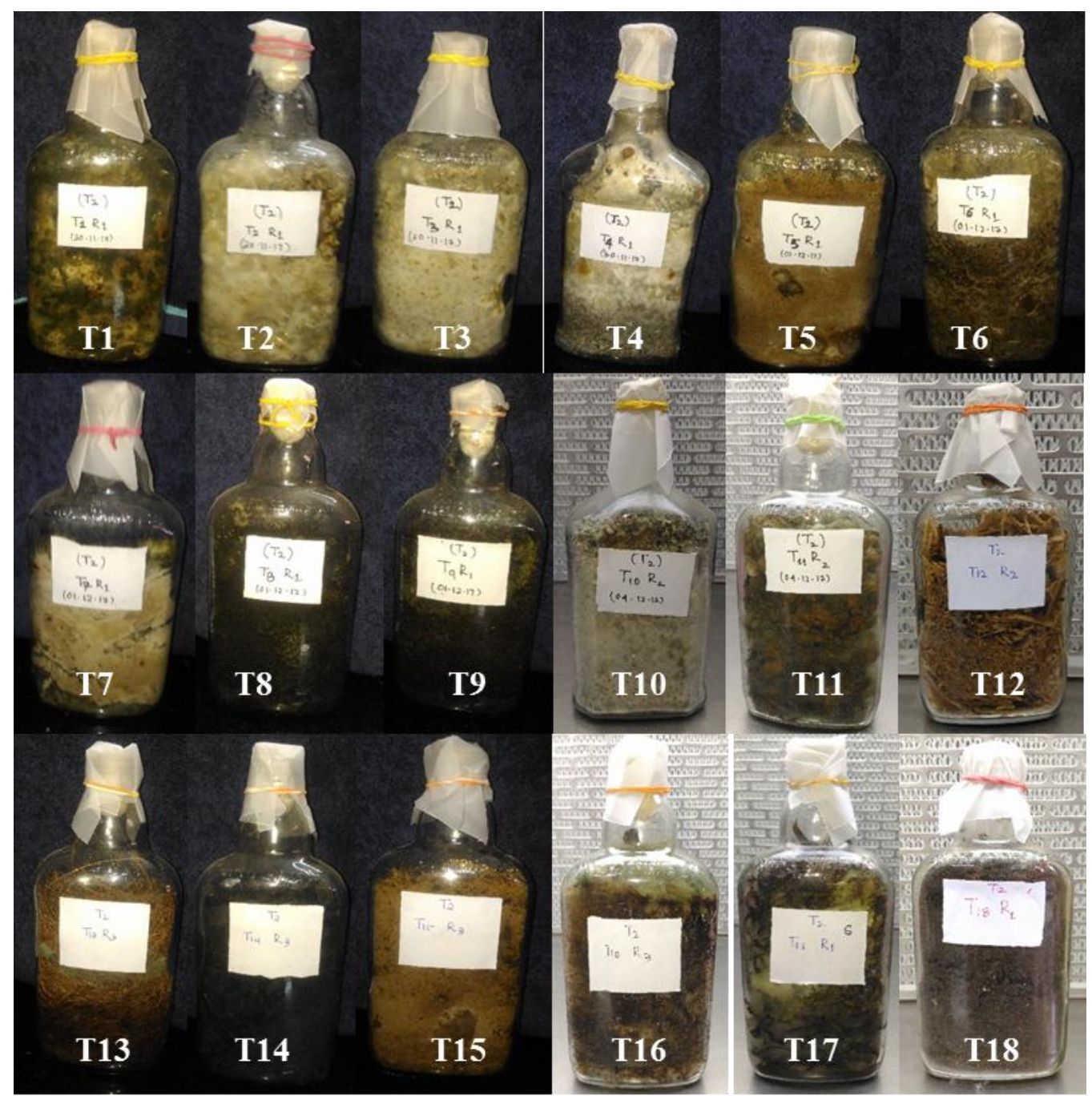

T1: Spent maize cob

T2: Maize grian

T3: Wheat grain

T4: Ragi grain

T5: Rice bran

T6: Paddy husk

T7: Cotton waste

T8: Farm yard Manure

T9: vermicompost
T10: Sorghum grain

T11: Groundnut shell

T12: Sugarcane bagasse

T13: Coir pith

T14: Charcoal

T15: Saw dust

T16: Spent mushroom substrate

T17: Pigeon pea husk

T18: Organic waste

Figure 2- Bottles containing different solid substrates incolulated with Trichoderma Isolate 5 

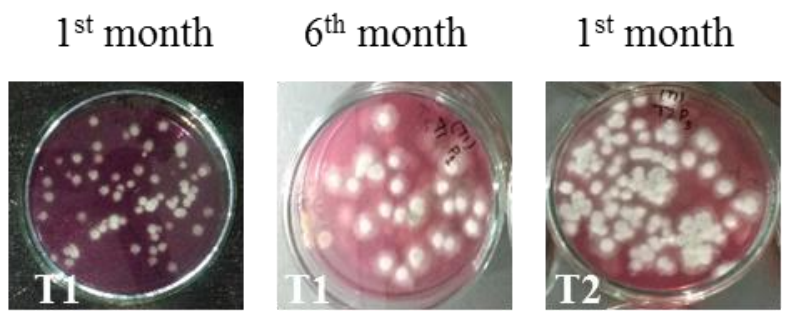

$6^{\text {th }}$ month
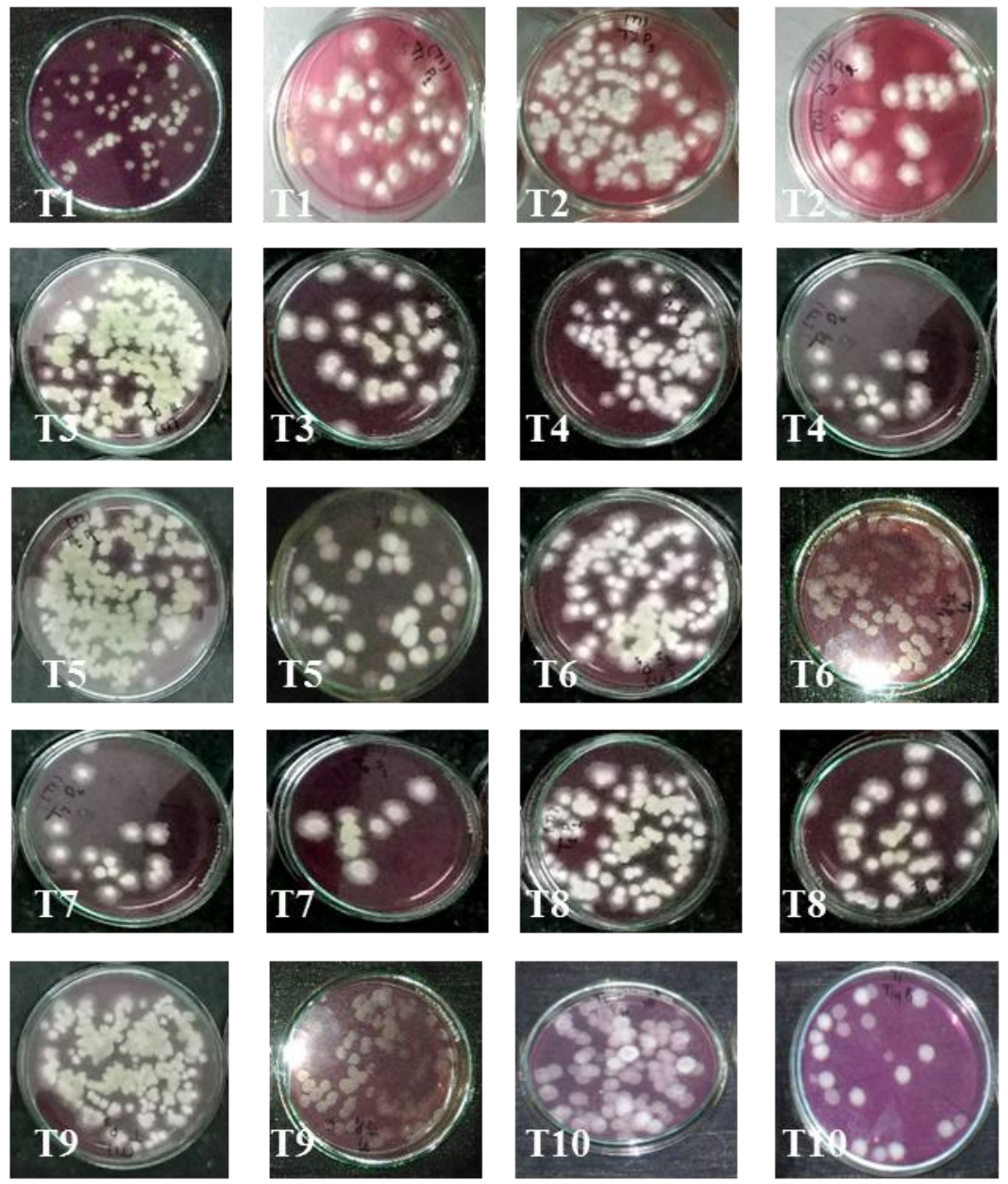

T1: Spent maize cob

T2: Maize grian

T3: Wheat grain

T4: Ragi grain

T5: Rice bran

T6: Paddy husk

T7: Cotton waste

T8: Farm yard Manure

T9: vermicompost

T10: Sorghum grain

Figure 3a-Petriplates showing colony forming units(cfu) of different solid substrates inoculated with Trichoderma Isolate 2 (T1-T10) 
$1^{\text {st }}$ month $\quad 6^{\text {th }}$ month $\quad 1^{\text {st }}$ month $\quad 6^{\text {th }}$ month
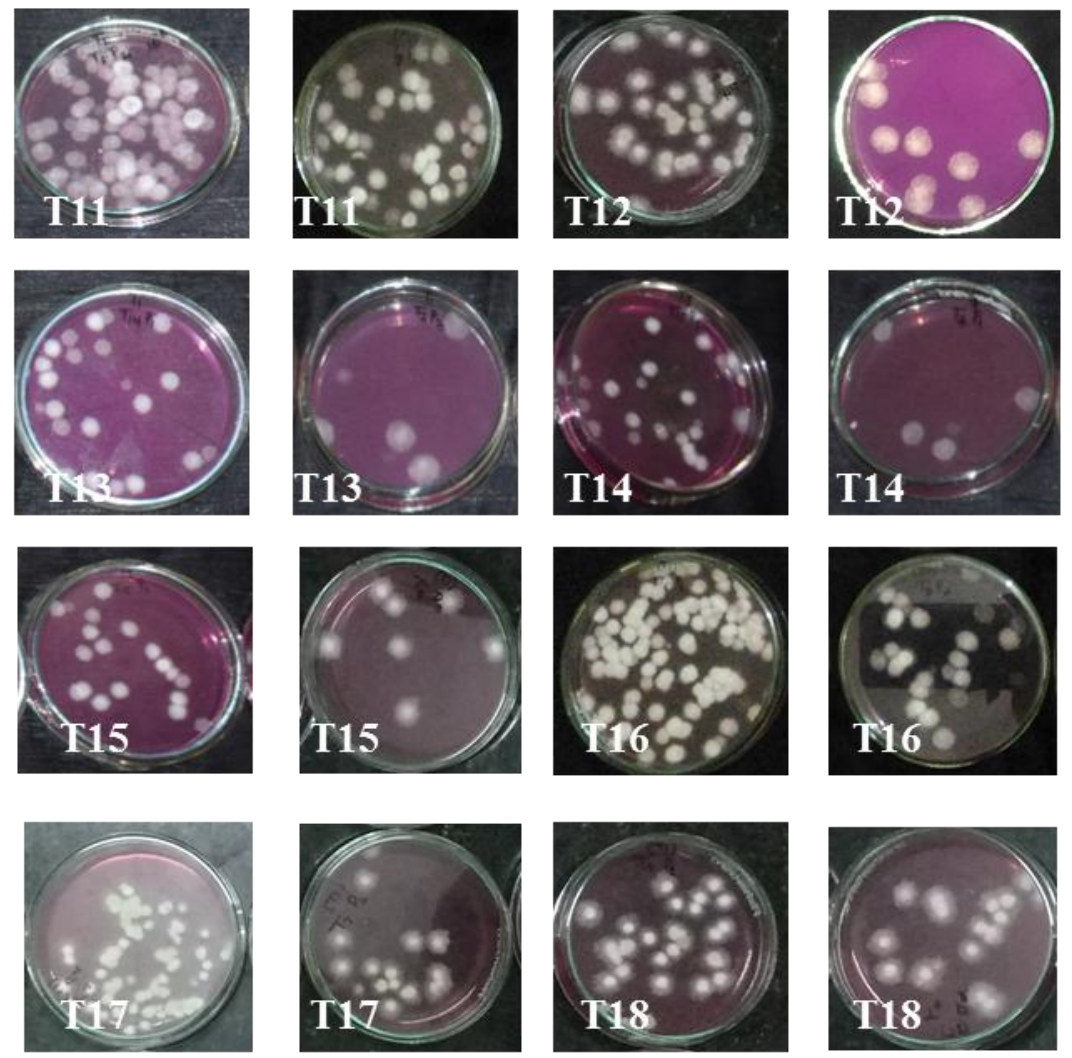

T11: Groundnut shell

T12: Sugarcane bagasse

T13: Coir pith

T14: Charcoal

T15: Saw dust

T16: Spent mushroom substrate

T17: Pigeon pea husk

T18: Organic waste

Figure 3b - Petriplates showing colony forming units of different solid substrates inoculated with Trichoderma Isolate 2 (T11-T18) 

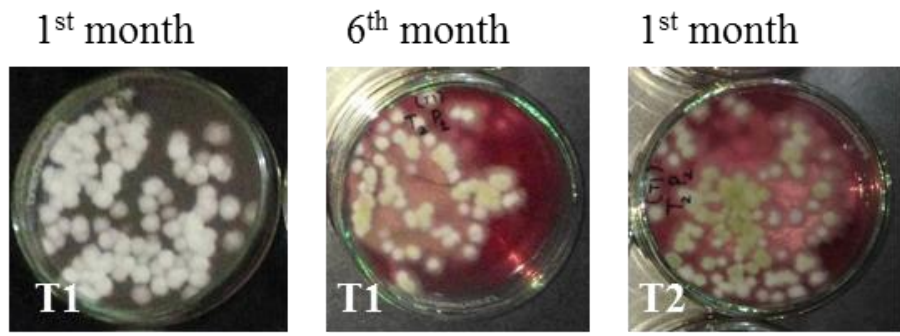

$6^{\text {th }}$ month
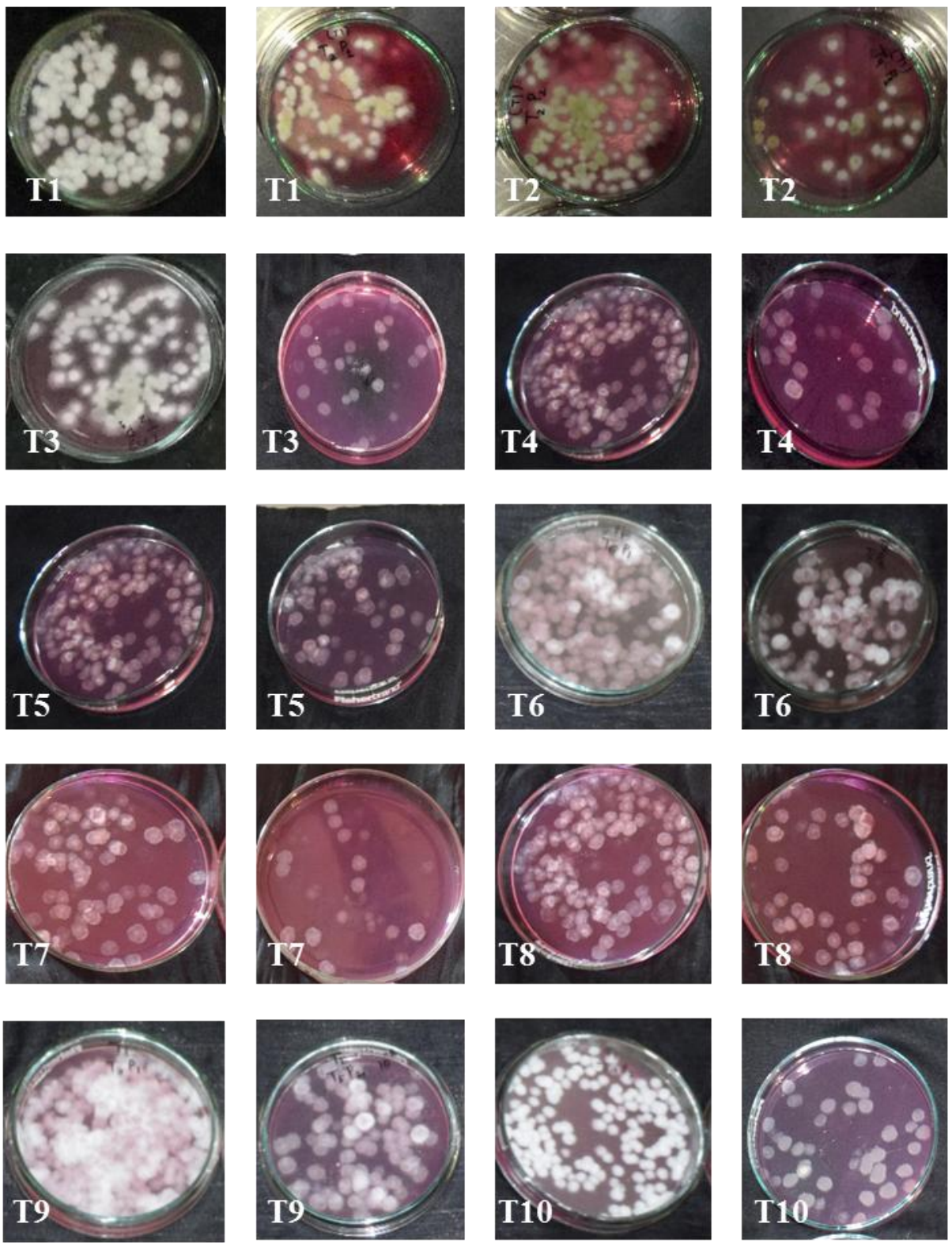

T1: Spent maize cob

T2: Maize grian

T3: Wheat grain

T4: Ragi grain

T5: Rice bran

T6: Paddy husk

T7: Cotton waste

T8: Farm yard Manure

T9: vermicompost

T10: Sorghum grain

Figure 4a - Petriplates showing colony forming units of different solid substrates inoculated with Trichoderma Isolate 5 (T1-T10) 

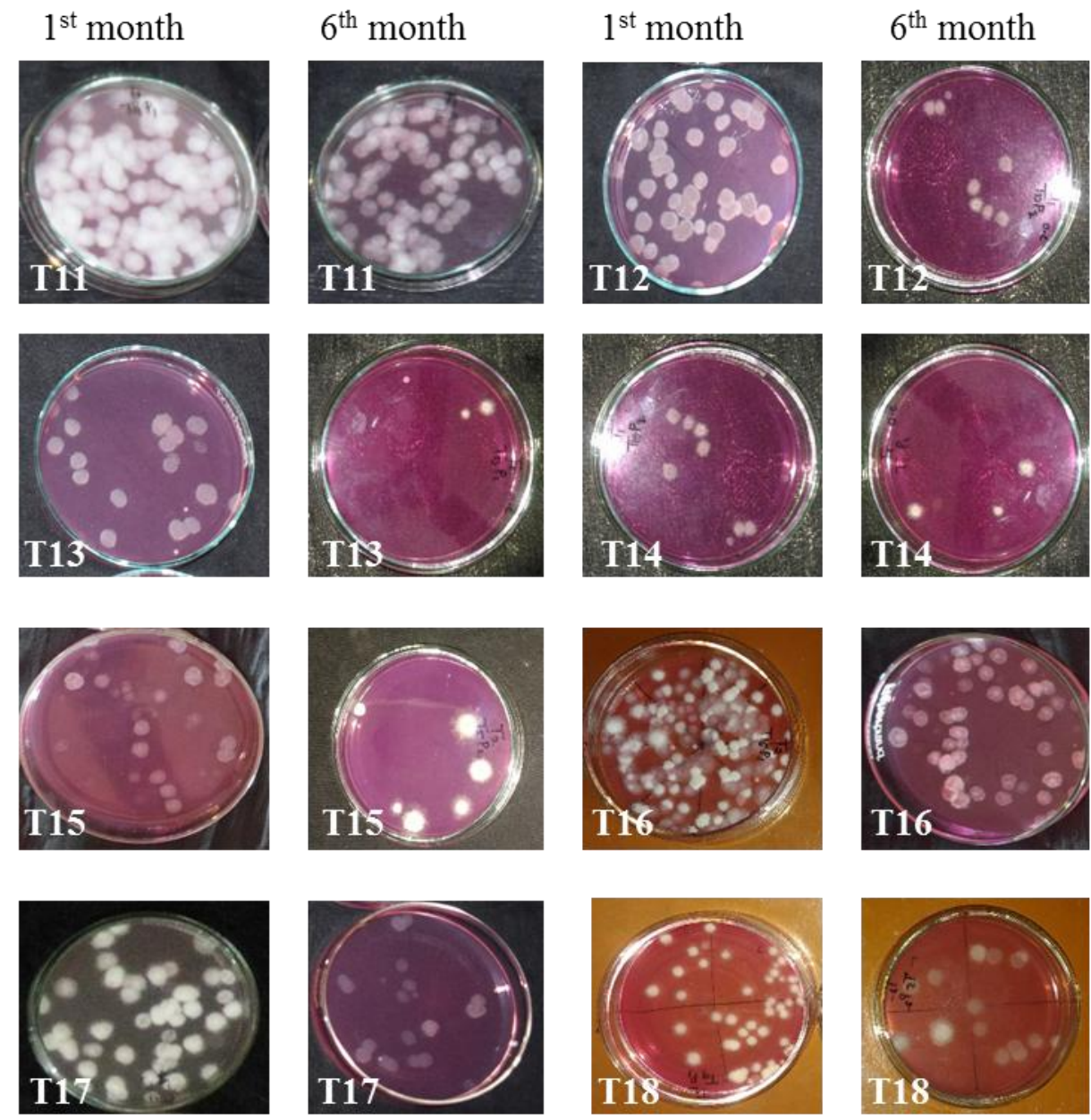

T11: Groundnut shell

T12: Sugarcane bagasse

T13: Coir pith

T14: Charcoal

T15: Saw dust

T16: Spent mushroom substrate

T17: Pigeon pea husk

T18: Organic waste

Figure 4b- Petriplates showing colony forming units of different solid substrates inoculated with Trichoderma Isolate 2 (T11-T18)

This experimental finding is found in compliance with the finding of Khan et al., (2011) where they used deoiled castor cake, gypsum, talc powder, vermicompost and well decomposed farmyard manure for preparation of $T$. viride formulation.
For Isolate 5, groundnut shell recorded to be the best performer with maximum cfu of $467.67 \times 10^{7} \mathrm{cfu} / \mathrm{g}$ in the first month followed by $332.00 \times 10^{7} \mathrm{cfu} / \mathrm{g}$ and $282.67 \times 10^{7} \mathrm{cfu} / \mathrm{g}$ in the second and third month respectively and the least cfu count $144.00 \times 10^{7} \mathrm{cfu} / \mathrm{g}$ was registered in the sixth month (Table-2, Figure- 
4a \& 4b), which is in agreement with findings of Kousalya et al.,(1988) who reported several substrates along with procedure for mass multiplication of antagonistic fungi and mentioned that, tapioca rind, well decomposed farm yard manure (FYM), well decomposed press mud, gobar gas slurry, mushroom spent bed, paddy husk, wheat bran and groundnut shell were found to be superior substrates for mass multiplication of $T$. harzianum. Therefore the effective substrates may be exploited for commercial production of Trichoderma sp.

\section{References}

Jeyarajan R. 2006. Prospects of indigenous mass production and formulation of Trichroderma, In Current Status of Biological Control of Plant diseases using antagonistic organisms in India (Eds Rabindra RJ Ramanujam B), pp. 74-80, 445, Project Directorate of Biological Control, Bangalore,.
Kousalya Gangadharan and Jayarajan R.1988. Techniques of mass multiplication of Trichoderma viride Pers ex. Fr. and Trichoderma harzianum Rifai. Nation Sem. Mange. Crop Dis. With Pl. Prod./Bio. Agents., pp, 32-33 Agriculture College and Research Institute, Tamil Nadu Agric. Univ., Madurai,

Kumar S and Gupta OM. 2012. Expanding dimension of Plant Pathology, JNKVV Research Journal, 6(3): 286-93.

Lewis JA. 1991. Formulation and delivery system of biocontrol agents with emphasis on fungi Beltsville symposia, The rhizosphere and plant growth (Keister DL and Cregan PB eds.), Agricultural Research 14:279-287.

Khan S, Bagwan NB, Iqbal MA and Tamboli RR. 2011. Mass Multiplication and Shelf life of Liquid Fermented final Product of Trichoderma viride in different formulations, Advances In Bioresearch, 2: 178 - 182.

\section{How to cite this article:}

Boblina, B., S. K. Beura, M. K. Mishra and Panda, A. G. 2019. Growth of Trichoderma spp on Different Solid Substrates. Int.J.Curr.Microbiol.App.Sci. 8(09): 2519-2529. doi: https://doi.org/10.20546/ijcmas.2019.809.292 